\begin{tabular}{|c|l|}
\hline Title & Sharp estimates for the Green function, 3G inequal ities, and nonlinear schrödinger problems in uniform cones \\
\hline Author(s) & Hirata, Kentaro \\
\hline Citation & Hokkaido University Preprint Series in Mathematics, 735, 1-18 \\
\hline Issue Date & 2005 \\
\hline DOI & 10.14943/83885 \\
\hline Doc URL & http://hdl.handle.net/2115/69543 \\
\hline Type & bulletin (article) \\
\hline File Information & pre735.pdf \\
\hline
\end{tabular}

Instructions for use 


\title{
SHARP ESTIMATES FOR THE GREEN FUNCTION, 3G INEQUALITIES, AND NONLINEAR SCHRÖDINGER PROBLEMS IN UNIFORM CONES
}

\author{
KENTARO HIRATA
}

\begin{abstract}
We find and prove sharp estimates for the Green function and 3G inequalities in uniform cones. Estimates are applied to give equivalent conditions for measures to satisfy the generalized Cranston-McConnell inequality, and to show the existence of infinitely many continuous solutions to nonlinear Schrödinger problems.
\end{abstract}

\section{Introduction}

We work in the Euclidean space $\mathbb{R}^{n}$, where $n \geq 3$. By $G_{\Omega}$, we denote the Green function for a domain $\Omega$, that is, for each $y \in \Omega$, the function $G_{\Omega}(\cdot, y)$ is the distributional solution to $-\Delta f=\delta_{y}$ in $\Omega$ and $f=0$ at all Dirichlet regular boundary points of $\Omega$. We write $\delta_{\Omega}(x)$ for the distance from $x \in \Omega$ to the Euclidean boundary $\partial \Omega$ of $\Omega$. By the symbol $A$, we denote an absolute positive constant whose value is unimportant and may change from line to line. If necessary, we use $A_{0}, A_{1}, \cdots$ to specify them. For two positive functions $f_{1}$ and $f_{2}$, we write $f_{1} \approx f_{2}$ if there exists a constant $A \geq 1$ such that $A^{-1} f_{1} \leq f_{2} \leq A f_{1}$. The constant $A$ will be called the constant of comparison.

The first purpose of the present paper is to show $3 \mathrm{G}$ inequalities in a cone by deriving a sharp global estimate for the Green function. In [6], Bogdan proved in a bounded Lipschitz domain $\Omega$ that if we fix $x_{0} \in \Omega$ and let $g(x)=\min \left\{1, G_{\Omega}\left(x, x_{0}\right)\right\}$, then

$$
G_{\Omega}(x, y) \approx \frac{g(x) g(y)}{g(b)^{2}}|x-y|^{2-n} \quad \text { for } x, y \in \Omega \text { and } b \in \mathcal{B}_{0}(x, y),
$$

where $\mathcal{B}_{0}(x, y)$ is, roughly speaking, the set of points $b$ in $\Omega$ that lie between $x$ and $y$ and satisfy $\delta_{\Omega}(b) \approx \max \left\{\delta_{\Omega}(x), \delta_{\Omega}(y),|x-y|\right\}$. See [6, p. 328] or Section 3 for the precise definition. Estimates of such a kind will play important roles when we treat the Green function. In fact, the following $3 \mathrm{G}$ inequality can be shown from the estimate (1.1). There exists a constant $A$ such that

$$
\frac{G_{\Omega}(x, y) G_{\Omega}(y, z)}{G_{\Omega}(x, z)} \leq A\left(|x-y|^{2-n}+|y-z|^{2-n}\right) \quad \text { for } x, y, z \in \Omega .
$$

Before the estimate (1.1), the 3G inequality was proved by Cranston, Fabes and Zhao [7] to study the conditional gauge theory for the Schrödinger operator. Recently, Aikawa and Lundh [4] extended (1.2) to a bounded uniformly John domain. The constants appearing in the estimates (1.1) and (1.2) depend on the diameter of a domain, and it seems that there is no results such as (1.1) and (1.2) in "unbounded" domains with no explicit expressions of the Green functions. We shall find and establish a sharp global

Key words and phrases. Green function, Martin kernel, 3G inequality, Cranston-McConnell inequality, Kato class, nonlinear Schrödinger equation

2000 Mathematics Subject Classification. Primary 31B35. Secondary 35J60. 
estimate for the Green function and 3G inequalities in particular unbounded domains, cones. We note that unbounded domains do not have (1.1) in general. For instance, considering the half space $\Omega=\left\{\left(x_{1}, \cdots, x_{n}\right): x_{n}>0\right\}$ and $x_{0}=(0, \cdots, 0,1)$, we see that $G_{\Omega}\left(r x_{0}, x_{0}\right) \approx r^{1-n}$ and $g\left(r x_{0}\right) g\left(x_{0}\right) g\left(b_{r}\right)^{-2}\left|r x_{0}-x_{0}\right|^{2-n} \approx g\left(r x_{0}\right)^{-1} r^{2-n} \approx r$ for $r>0$ sufficiently large and $b_{r} \in \mathcal{B}\left(r x_{0}, x_{0}\right)$. Therefore it is interesting to find a sharp global estimate for the Green function in a cone. Indeed, we will establish (1.1) using the Martin kernel at infinity instead of $g$. Our results will be stated in Section 3. As one of interesting applications of a $3 \mathrm{G}$ inequality, we shall give equivalent conditions for measures $\nu$ to satisfy the generalized Cranston-McConnell inequality:

$$
\int_{\Omega} G_{\Omega}(x, y) u(y) d \nu(y) \leq A u(x)
$$

for all $x \in \Omega$ and all positive superharmonic functions $u$ in $\Omega$. We will see suprisingly that if this inequality holds only for the Martin kernel at infinity, then one holds for all positive superharmonic functions.

The second purpose is to show the existence of infinitely many continuous solutions to the following nonlinear Schrödinger problem in a cone $\Omega$ :

$$
\left\{\begin{array}{l}
\Delta u-\mu u=f(\cdot, u) \text { in } \Omega \text { (in the sense of distributions), } \\
u>0 \text { in } \Omega \\
u=0 \text { on } \partial_{r} \Omega,
\end{array}\right.
$$

where $\mu$ and $f$ are respectively a signed measure on $\Omega$ and a Borel measurable function in $\Omega \times(0,+\infty)$ with suitable properties stated in Section 5 , and $\partial_{r} \Omega$ is the set of all Dirichlet regular points of $\partial \Omega$. Zhang and Zhao [11] studied the problem (1.3) with $\mu=0$ in a bounded Lipschitz domain containing the origin and showed, using the $3 \mathrm{G}$ inequality (1.2), the existence of singular solutions with the growth $|\cdot|^{2-n}$ near the origin. The existence of bounded solutions in an unbounded domain with a compact Lipschitz boundary was investigated in [12]. In [5], Bachar, Mâagli and Zribi studied the problem (1.3) with $\mu=0$ in the half space and showed the existence of solutions with the growth $x_{n}$ near infinity. Their discussion was based on the explicit expression of the Green function. Thus our purpose is to extend their result to cones by applying our sharp estimates for the Green function. In particular, we shall show the existence of solutions with the same growth as the Martin kernel at infinity.

The following notations will be used in this paper. By $B(x, r)$ and $S(x, r)$, we denote the open ball and the sphere of center $x$ and radius $r$, respectively. When $x$ is the origin, we write $B(r)=B(x, r)$ and $S(r)=S(x, r)$ for simplicity. A cone we consider is an unbounded domain of the form

$$
\Gamma=\left\{x \in \mathbb{R}^{n} \backslash\{0\}: \frac{x}{|x|} \in \omega\right\},
$$

where $\omega$ is some relatively open subset of $S(1)$. In particular, we will consider uniform cones. See Section 2.

The plan of this paper is as follows. In Section 2, we shall collect definitions of a uniform cone and the Martin kernel, and give elementary and useful properties. We also state our key tools: the Carleson estimate and the boundary Harnack principle. In Section 3, we shall establish a sharp global estimate for the Green function in a uniform cone, and show new and classical 3G inequalities. Also, other inequalities that used 
in subsequent sections will be proved. In Section 4, we shall give a characterization of measures that enjoy the generalized Cranston-McConnell inequality, as an application of a New 3G inequality. We also introduce a certain class of measures which is bigger than the classical Kato class, and give some properties. In Section 5, we investigate the problem (1.3) in a uniform cone.

\section{Preliminaries}

2.1. Uniform cones. We first state the definition of a uniform cone. A cone $\Gamma$ in $\mathbb{R}^{n}$ is said to be a uniform cone if there exists a constant $A_{0} \geq 1$ such that each pair of points $x$ and $y$ in $\Gamma \cap B(1)$ can be connected by a rectifiable curve $\gamma$ in $\Gamma$ for which

$$
\begin{aligned}
& \ell(\gamma) \leq A_{0}|x-y|, \\
& \min \{\ell(\gamma(x, z)), \ell(\gamma(z, y))\} \leq A_{0} \delta_{\Gamma}(z) \quad \text { for all } z \in \gamma,
\end{aligned}
$$

where $\ell(\gamma(x, z))$ denotes the length of the subarc $\gamma(x, z)$ of $\gamma$ from $x$ to $z$, and $\delta_{\Gamma}(z)$ stands for the distance from $z$ to $\partial \Gamma$. We note that a uniform cone is a uniform domain in the sense of [9].

Lemma 2.1. If $\Gamma$ is a uniform cone, then each pair of points $x$ and $y$ in $\Gamma$ can be connected by a rectifiable curve $\gamma$ in $\Gamma$ satisfying (2.1) with the same constant $A_{0}$.

Proof. Let $x, y \in \Gamma$ and let $r>\max \{|x|,|y|\}$. Then $x / r, y / r \in \Gamma \cap B(1)$. By assumption, there is a curve $\gamma$ in $\Gamma$ connecting $x / r$ to $y / r$ with the property (2.1) for $x / r$ and $y / r$ in place of $x$ and $y$. Let $\gamma_{r}=\{r z: z \in \gamma\}$. Then $\gamma_{r}$ is a curve in $\Gamma$ connecting $x$ to $y$. It also follows that $\ell\left(\gamma_{r}\right)=r \ell(\gamma) \leq A_{0}|x-y|$ and that for $w \in \gamma_{r}$,

$$
\begin{aligned}
\min \left\{\ell\left(\gamma_{r}(x, w)\right), \ell\left(\gamma_{r}(w, y)\right)\right\} & =r \min \{\ell(\gamma(x / r, w / r)), \ell(\gamma(w / r, y / r))\} \\
& \leq r A_{0} \delta_{\Gamma}(w / r)=A_{0} \delta_{\Gamma}(w) .
\end{aligned}
$$

Thus the lemma is proved.

2.2. Quasi-hyperbolic metric and Harnack inequality. We state the Harnack inequality involving the quasi-hyperbolic metric in a uniform cone. The quasi-hyperbolic metric on $\Gamma$ is defined by

$$
k_{\Gamma}(x, y)=\inf _{\gamma} \int_{\gamma} \frac{d s(z)}{\delta_{\Gamma}(z)}
$$

where the infimum is taken over all rectifiable curves $\gamma$ in $\Gamma$ connecting $x$ to $y$, and $d s$ stands for the line element on $\gamma$. Note from [9] that a uniform cone is characterized in terms of the quasi-hyperbolic metric:

$$
k_{\Gamma}(x, y) \leq A \log \left[\left(\frac{|x-y|}{\delta_{\Gamma}(x)}+1\right)\left(\frac{|x-y|}{\delta_{\Gamma}(y)}+1\right)\right]+A \quad \text { for } x, y \in \Gamma .
$$

We also note from [3, Lemma 7.2] that if $z \in \Gamma$, then

$$
k_{\Gamma \backslash\{z\}}(x, y) \leq 3 k_{\Gamma}(x, y)+\pi \quad \text { for } x, y \in \Gamma \backslash B\left(z, 2^{-1} \delta_{\Gamma}(z)\right) .
$$

A finite sequence of balls $\left\{B\left(x_{j}, 2^{-1} \delta_{\Gamma}\left(x_{j}\right)\right)\right\}_{j=1}^{M}$ in $\Gamma$ is called a Harnack chain of length $M$ joining $x$ and $y$ if $x_{1}=x, x_{M}=y$, and $x_{j+1} \in B\left(x_{j}, 2^{-1} \delta_{\Gamma}\left(x_{j}\right)\right)$ for $j=1, \cdots, M-1$. 
We observe that the shortest length of the Harnack chain joining $x$ and $y$ is comparable to $k_{\Gamma}(x, y)+1$. Therefore there exists a constant $A \geq 1$ depending only on $\Gamma$ such that

$$
\exp \left(-A\left(k_{\Gamma}(x, y)+1\right)\right) \leq \frac{h(x)}{h(y)} \leq \exp \left(A\left(k_{\Gamma}(x, y)+1\right)\right) \quad \text { for } x, y \in \Gamma,
$$

whenever $h$ is a positive harmonic function in $\Gamma$. As a consequence, we can obtain the following lemma.

Lemma 2.2. Let $\Gamma$ be a uniform cone, and let $h$ be a positive harmonic function in $\Gamma$. If $x, y \in \Gamma$ satisfy $|x-y| \leq A_{3} \min \left\{\delta_{\Gamma}(x), \delta_{\Gamma}(y)\right\}$ for some $A_{3}>0$, then

$$
h(x) \approx h(y) \text { and } G_{\Gamma}(x, y) \approx|x-y|^{2-n},
$$

where the constants of comparisons depend only on $A_{3}$ and $\Gamma$.

2.3. Carleson estimate and Boundary Harnack principle. We next state the Carleson estimate and the boundary Harnack principle. We say that a property holds quasi-everywhere if it holds apart from a polar set. The following lemma is found in $[2$, Theorem 1 and Remark 2].

Lemma 2.3. Let $\Gamma$ be a uniform cone. Then there exist positive constants $r_{1}$ and $A_{1}<1$ depending only on $\Gamma$ with the following property: Let $\xi \in \partial \Gamma$ and $0<r \leq r_{1}$. If $h_{1}$ and $h_{2}$ are positive bounded harmonic functions in $\Gamma \cap B(\xi, r)$ vanishing quasieverywhere on $\partial \Gamma \cap B(\xi, r)$, then

$$
\frac{h_{1}(x)}{h_{2}(x)} \approx \frac{h_{1}\left(x^{\prime}\right)}{h_{2}\left(x^{\prime}\right)} \quad \text { for } x, x^{\prime} \in \Gamma \cap \overline{B\left(\xi, A_{1} r\right)},
$$

where the constant of comparison depends only on $\Gamma$. Moreover, if $z$ is an arbitrary point in $\Gamma \cap S\left(\xi, A_{1} r\right)$ such that $\delta_{\Gamma}(z) \geq A_{2} r$ for some $A_{2}>0$, then

$$
h_{1}(x) \leq A h_{1}(z) \quad \text { for } x \in \Gamma \cap \overline{B\left(\xi, A_{1} r\right)},
$$

where the constant $A$ depends only on $A_{2}$ and $\Gamma$.

Remark 2.4. In arguments below, a constant $A_{2}$ in Lemma 2.3 will be implicitly taken as $2^{-1} A_{0}^{-1} A_{1}$. The existence of a point $z \in \Gamma \cap S\left(\xi, A_{1} r\right)$ with $\delta_{\Gamma}(z) \geq 2^{-1} A_{0}^{-1} A_{1} r$ can be shown as follows. Let $x \in \Gamma \cap S\left(\xi, 2^{-1} A_{1} r\right)$ and $y \in \Gamma \cap S\left(\xi, 2 A_{1} r\right)$. By Lemma 2.1, there exists a curve $\gamma$ in $\Gamma$ connecting $x$ to $y$ with the properties in (2.1). Then a point $z \in \gamma \cap S\left(\xi, A_{1} r\right)$ satisfies $\delta_{\Gamma}(z) \geq 2^{-1} A_{0}^{-1} A_{1} r$.

2.4. Martin kernels. We finally state the definition of the Martin kernels. Let $\Omega$ be a unbounded domain in $\mathbb{R}^{n}$. Recall that $G_{\Omega}$ is the Green function for $\Omega$. We fix $x_{0} \in \Omega$ (the reference point). Let $\xi \in \partial \Omega \cup\{\infty\}$, and let $\left\{y_{j}\right\}$ be a sequence in $\Omega$ converging to $\xi$. Then we see that some subsequence of $\left\{G_{\Omega}\left(\cdot, y_{j}\right) / G_{\Omega}\left(x_{0}, y_{j}\right)\right\}_{j}$ converges to a positive harmonic function in $\Omega$. All limit functions obtained in this way are called Martin kernels at $\xi$. When we consider a cone $\Gamma$, the reference point $x_{0}$ is taken in $\Gamma \cap S(1)$.

Lemma 2.5. If $\Gamma$ is a uniform cone, then for each $\xi \in \partial \Gamma \cup\{\infty\}$, there exists a unique (minimal) Martin kernel $K_{\Gamma}(\cdot, \xi)$ at $\xi$. Moreover, there exist a non-negative constant $\alpha$ and a positive bounded continuous function $\theta$ on $\Gamma \cap S(1)$ such that

$$
K_{\Gamma}(x, 0)=|x|^{2-n-\alpha} \theta(x /|x|) \quad \text { and } \quad K_{\Gamma}(x, \infty)=|x|^{\alpha} \theta(x /|x|) .
$$


Proof. The first assertion for $\xi \in \partial \Gamma$ is found in [2, Theorem 3]. By the Kelvin transform, we also observe that there is a unique (minimal) Martin kernel at $\infty$. The representation (2.4) can be obtained by the similar way as in [10, p. 472].

It is noteworthy that if $\Gamma$ is a uniform cone, then for $r>0$,

$$
G_{\Gamma}(x, y)=r^{2-n} G_{\Gamma}(x / r, y / r) \quad \text { and } \quad K_{\Gamma}(x, \infty)=r^{\alpha} K_{\Gamma}(x / r, \infty) .
$$

We also see from (2.2) and (2.3) that there exist positive constants $A$ and $\beta \geq 1$ such that

$$
\theta(z) \geq A \delta_{\Gamma}(z)^{\beta} \quad \text { for } z \in \Gamma \cap S(1) .
$$

Note that if $\Gamma \cap S(1)$ has a $C^{1,1}$-boundary, then we can take $\beta=1$.

\section{Sharp estimates for the Green function and 3G inequalities}

Throughout this section, we suppose that $\Gamma$ is a uniform cone in $\mathbb{R}^{n}$ with $n \geq 3$. To obtain the estimate (1.1) in a bounded Lipschitz domain $\Omega$, Bogdan [6] defined $\mathcal{B}_{0}(x, y)$ as the set of all points $b$ in $\Omega$ such that $B\left(b, \kappa_{0} r\right) \subset \Omega \cap B(x, 3 r) \cap B(y, 3 r)$ if $r:=\max \left\{\delta_{\Omega}(x), \delta_{\Omega}(y),|x-y|\right\} \leq r_{2}$, and $\mathcal{B}_{0}(x, y)=S\left(x_{0}, r_{2}\right)$ if $r>r_{2}$, where $\kappa_{0}$ and $r_{2}$ are some fixed positive constants. We know that $\mathcal{B}_{0}(x, y)$ plays a good role essentially when $\delta_{\Omega}(x)$ and $\delta_{\Omega}(y)$ are much smaller than $|x-y|$. From this view, we adopt the following somewhat simpler definition. Let $\kappa \geq 1$. For $x, y \in \Gamma$, we define

$$
\mathcal{B}(x, y)=\left\{b \in \Gamma: \max \{|x-b|,|b-y|\} \leq \kappa|x-y| \text { and } \delta_{\Gamma}(b) \geq \frac{|x-y|}{\kappa}\right\} .
$$

Although this definition does not include the relation between $\delta_{\Gamma}(b)$ and $\max \left\{\delta_{\Gamma}(x), \delta_{\Gamma}(y)\right\}$, we have the following.

Proposition 3.1. Let $x, y \in \Gamma$, and let $A_{0}$ be the constant in (2.1). The following statements hold.

(i) If $\kappa \geq 2 A_{0}$, then $\mathcal{B}(x, y)$ is non-empty, and $\mathcal{B}(x, y)=\mathcal{B}(y, x)$.

(ii) If $b \in \mathcal{B}(x, y)$, then $\delta_{\Gamma}(b) \geq\left(2 \kappa^{2}\right)^{-1} \max \left\{\delta_{\Gamma}(x), \delta_{\Gamma}(y)\right\}$ and $|b| \geq\left(2 \kappa^{2}\right)^{-1} \max \{|x|,|y|\}$.

(iii) If $r>0$, then $\mathcal{B}(r x, r y)=\{b \in \Gamma: b / r \in \mathcal{B}(x, y)\}$.

Proof. (i) Let $x, y \in \Gamma$. By Lemma 2.1, there exists a curve $\gamma$ in $\Gamma$ connecting $x$ to $y$ such that $\ell(\gamma) \leq A_{0}|x-y|$ and $\min \{|x-z|,|z-y|\} \leq A_{0} \delta_{\Gamma}(z)$ for all $z \in \gamma$. Let $b$ be a point in $\gamma$ such that $|x-b|=|b-y|$. Then

$$
\begin{aligned}
& \max \{|x-b|,|b-y|\} \leq \ell(\gamma) \leq A_{0}|x-y|, \\
& \delta_{\Gamma}(b) \geq A_{0}^{-1}|x-b| \geq\left(2 A_{0}\right)^{-1}|x-y| .
\end{aligned}
$$

Hence $\mathcal{B}(x, y)$ is non-empty whenever $\kappa \geq 2 A_{0}$. The symmetry of $\mathcal{B}(x, y)$ is clear from the definition.

(ii) We first show $\delta_{\Gamma}(b) \geq\left(2 \kappa^{2}\right)^{-1} \max \left\{\delta_{\Gamma}(x), \delta_{\Gamma}(y)\right\}$. By symmetry, it suffices to prove $\delta_{\Gamma}(b) \geq\left(2 \kappa^{2}\right)^{-1} \delta_{\Gamma}(x)$. Suppose to the contrary that there is $b \in \mathcal{B}(x, y)$ such that $\delta_{\Gamma}(b)<\left(2 \kappa^{2}\right)^{-1} \delta_{\Gamma}(x)$. Then $|x-y| \leq \kappa \delta_{\Gamma}(b) \leq(2 \kappa)^{-1} \delta_{\Gamma}(x)$, and so $|x-b| \leq \kappa|x-y| \leq$ $2^{-1} \delta_{\Gamma}(x)$. Hence

$$
\delta_{\Gamma}(b) \geq \delta_{\Gamma}(x)-|x-b| \geq 2^{-1} \delta_{\Gamma}(x) \geq\left(2 \kappa^{2}\right)^{-1} \delta_{\Gamma}(x) .
$$

This is a contradiction. We next show $|b| \geq\left(2 \kappa^{2}\right)^{-1} \max \{|x|,|y|\}$. It is enough to prove $|b| \geq\left(2 \kappa^{2}\right)^{-1}|x|$. Suppose to the contrary that there is $b \in \mathcal{B}(x, y)$ such that 
$|b|<\left(2 \kappa^{2}\right)^{-1}|x|$. Then $|x-y| \leq(2 \kappa)^{-1}|x|$ since $\delta_{\Gamma}(b) \leq|b|$, and so $|x-b| \leq 2^{-1}|x|$. Hence

$$
|b| \geq|x|-|x-b| \geq 2^{-1}|x| \geq(2 \kappa)^{-1}|x| .
$$

This is a contradiction.

(iii) Since $\delta_{\Gamma}(r b)=r \delta_{\Gamma}(b)$ and $|r x-r y|=r|x-y|$, we can obtain (iii) immediately.

The sharp global estimate for the Green function in $\Gamma$ is as follows.

Theorem 3.2. For $x, y \in \Gamma$ and $b \in \mathcal{B}(x, y)$,

$$
G_{\Gamma}(x, y) \approx \frac{K_{\Gamma}(x, \infty) K_{\Gamma}(y, \infty)}{K_{\Gamma}(b, \infty)^{2}}|x-y|^{2-n},
$$

where the constant of comparison depends only on $\kappa$ and $\Gamma$.

Proof. We first show (3.1) for $x, y \in \Gamma \cap B(1)$ and $b \in \mathcal{B}(x, y)$. We may assume by symmetry that $\delta_{\Gamma}(x) \leq \delta_{\Gamma}(y)$. Let $A_{4}=A_{1}^{-1} \max \left\{5,2 r_{1}^{-1}\right\}$, where $0<A_{1}<1$ and $r_{1}>0$ are the constants in Lemma 2.3. We consider two cases: $|x-y| \leq A_{4} \delta_{\Gamma}(x)$ and $|x-y|>A_{4} \delta_{\Gamma}(x)$.

Case 1: $|x-y| \leq A_{4} \delta_{\Gamma}(x)$. Since $|x-b| \leq \kappa|x-y| \leq \kappa^{2} \delta_{\Gamma}(b)$ by the definition of $\mathcal{B}(x, y)$, it follows from Lemma 2.2 that

$$
K_{\Gamma}(b, \infty) \approx K_{\Gamma}(x, \infty) \approx K_{\Gamma}(y, \infty) \text { and } G_{\Gamma}(x, y) \approx|x-y|^{2-n} .
$$

Hence we obtain (3.1) in this case.

Case 2: $|x-y|>A_{4} \delta_{\Gamma}(x)$. Note from our choice of $A_{4}$ that

$$
A_{1}^{-1} A_{4}^{-1}|x-y| \leq 2 A_{1}^{-1} A_{4}^{-1} \leq r_{1} .
$$

Let $\xi \in \partial \Gamma$ be a point such that $\delta_{\Gamma}(x)=|x-\xi|$. Then

$$
|y-\xi| \geq|x-y|-|x-\xi| \geq\left(1-A_{4}^{-1}\right)|x-y| \geq A_{1}^{-1} A_{4}^{-1}|x-y| .
$$

We take $x_{1} \in \Gamma \cap S\left(\xi, A_{4}^{-1}|x-y|\right)$ with $\delta_{\Gamma}\left(x_{1}\right) \approx|x-y|$ (cf. Remark 2.4). By Lemma 2.3, we have

$$
\frac{G_{\Gamma}(x, y)}{K_{\Gamma}(x, \infty)} \approx \frac{G_{\Gamma}\left(x_{1}, y\right)}{K_{\Gamma}\left(x_{1}, \infty\right)} .
$$

We take $y_{1}$ as follows. If $\delta_{\Gamma}(y) \geq A_{4}^{-1}|x-y|$, then we let $y_{1}=y$. If $\delta_{\Gamma}(y)<A_{4}^{-1}|x-y|$, then, letting $\eta \in \partial \Gamma$ be a point such that $\delta_{\Gamma}(y)=|y-\eta|$, we take $y_{1} \in \Gamma \cap S\left(\eta, A_{4}^{-1}|x-y|\right)$ with $\delta_{\Gamma}\left(y_{1}\right) \approx|x-y|$. Note in the latter case that

$$
\left|x_{1}-\eta\right| \geq|x-y|-\left|x-x_{1}\right|-|y-\eta| \geq\left(1-3 A_{4}^{-1}\right)|x-y| \geq A_{1}^{-1} A_{4}^{-1}|x-y| .
$$

We have by Lemma 2.3

$$
\frac{G_{\Gamma}\left(x_{1}, y\right)}{K_{\Gamma}(y, \infty)} \approx \frac{G_{\Gamma}\left(x_{1}, y_{1}\right)}{K_{\Gamma}\left(y_{1}, \infty\right)} .
$$

Since $\left|x_{1}-y_{1}\right| \approx|x-y| \leq A \min \left\{\delta_{\Gamma}\left(x_{1}\right), \delta_{\Gamma}\left(y_{1}\right)\right\}$, it follows from Case 1 that for $b_{1} \in \mathcal{B}\left(x_{1}, y_{1}\right)$,

$$
G_{\Gamma}\left(x_{1}, y_{1}\right) \approx \frac{K_{\Gamma}\left(x_{1}, \infty\right) K_{\Gamma}\left(y_{1}, \infty\right)}{K_{\Gamma}\left(b_{1}, \infty\right)^{2}}|x-y|^{2-n} .
$$

Note that $\delta_{\Gamma}\left(b_{1}\right) \geq \kappa^{-1}\left|x_{1}-y_{1}\right| \approx|x-y|$ and

$$
\left|b-b_{1}\right| \leq|b-x|+\left|x-x_{1}\right|+\left|x_{1}-b_{1}\right| \leq\left(\kappa+2 A_{4}^{-1}\right)|x-y|+\kappa\left|x_{1}-y_{1}\right| \approx|x-y|,
$$


and so $\left|b-b_{1}\right| \leq A \min \left\{\delta_{\Gamma}(b), \delta_{\Gamma}\left(b_{1}\right)\right\}$. Therefore Lemma 2.2 yields that

$$
K_{\Gamma}\left(b_{1}, \infty\right) \approx K_{\Gamma}(b, \infty) .
$$

Combining (3.2), (3.3), (3.4) and (3.5), we obtain (3.1) in this case. Thus (3.1) holds for $x, y \in \Gamma \cap B(1)$ and $b \in \mathcal{B}(x, y)$.

Finally, to establish (3.1) for all $x, y \in \Gamma$ and $b \in \mathcal{B}(x, y)$, we let $r>\max \{|x|,|y|\}$. Then $x / r, y / r \in \Gamma \cap B(1)$ and $b / r \in \mathcal{B}(x / r, y / r)$ by Proposition 3.1. Therefore we have from the above observation

$$
G_{\Gamma}(x / r, y / r) \approx \frac{K_{\Gamma}(x / r, \infty) K_{\Gamma}(y / r, \infty)}{K_{\Gamma}(b / r, \infty)^{2}}\left|\frac{x}{r}-\frac{y}{r}\right|^{2-n} .
$$

Hence (3.1) follows from (2.5). Thus the proof is complete.

In what follows, we take $\kappa=2 A_{0}$ but we continue to use the symbol $\kappa$. If $x$ and $y$ are separated enough, then the Green function is comparable to the product of the Martin kernels at the origin and at infinity.

Corollary 3.3. For $x, y \in \Gamma$ with $2|y| \leq|x|$,

$$
G_{\Gamma}(x, y) \approx K_{\Gamma}(x, 0) K_{\Gamma}(y, \infty),
$$

where the constant of comparison depends only on $\Gamma$.

Proof. Let $x, y \in \Gamma$ satisfy $2|y| \leq|x|$, and let $b \in \mathcal{B}(x, y)$. Then

$$
(2 \kappa)^{-1}|x| \leq \kappa^{-1}|x-y| \leq \delta_{\Gamma}(b) \leq|b| \leq|b-x|+|x| \leq \kappa|x-y|+|x| \leq 3 \kappa|x|,
$$

and so $\delta_{\Gamma}(b /|b|)=|b|^{-1} \delta_{\Gamma}(b) \geq\left(6 \kappa^{2}\right)^{-1}$. It follows from Lemma 2.5 that $K_{\Gamma}(b, \infty) \approx$ $|b|^{\alpha} \approx|x|^{\alpha}$. Hence we obtain from Theorem 3.2 and (2.4) that

$$
G_{\Gamma}(x, y) \approx K_{\Gamma}(x, \infty) K_{\Gamma}(y, \infty)|x|^{2-n-2 \alpha}=K_{\Gamma}(x, 0) K_{\Gamma}(y, \infty) .
$$

Thus the corollary follows.

As an important application of Theorem 3.2, we obtain the following $3 \mathrm{G}$ inequality.

Theorem 3.4 (New 3G inequality). There exists a constant $A$ depending only on $\Gamma$ such that for $x, y, z \in \Gamma$,

$$
\frac{G_{\Gamma}(x, y) G_{\Gamma}(y, z)}{G_{\Gamma}(x, z)} \leq A\left(\frac{K_{\Gamma}(y, \infty)}{K_{\Gamma}(x, \infty)} G_{\Gamma}(x, y)+\frac{K_{\Gamma}(y, \infty)}{K_{\Gamma}(z, \infty)} G_{\Gamma}(y, z)\right) .
$$

Proof. We may assume by (2.5) and symmetry that $x, y, z \in \Gamma \cap B(1)$ and $|x-y| \leq|y-z|$. It is enough to show that

$$
\frac{G_{\Gamma}(y, z)}{G_{\Gamma}(x, z)} \leq A \frac{K_{\Gamma}(y, \infty)}{K_{\Gamma}(x, \infty)} .
$$

Let $b_{x, z} \in \mathcal{B}(x, z)$ and $b_{y, z} \in \mathcal{B}(y, z)$. Since $|x-z| \leq|x-y|+|y-z| \leq 2|y-z|$, it follows from Theorem 3.2 that

$$
\frac{G_{\Gamma}(y, z)}{G_{\Gamma}(x, z)} \leq A\left(\frac{K_{\Gamma}\left(b_{x, z}, \infty\right)}{K_{\Gamma}\left(b_{y, z}, \infty\right)}\right)^{2} \frac{K_{\Gamma}(y, \infty)}{K_{\Gamma}(x, \infty)} .
$$

We claim that

$$
\frac{K_{\Gamma}\left(b_{x, z}, \infty\right)}{K_{\Gamma}\left(b_{y, z}, \infty\right)} \leq A
$$


To show this, we let $A_{5}=3 \kappa A_{1}^{-1}$ and $r=A_{5} \max \left\{|y-z|, \delta_{\Gamma}(z)\right\}$. We consider two cases: $r \geq r_{1}$ and $r<r_{1}$. Here $0<A_{1}<1$ and $r_{1}>0$ are the constants in Lemma 2.3.

Case 1: $r \geq r_{1}$. Since $\left|b_{x, z}\right| \leq 1+2 \kappa$, it follows from Lemma 2.5 that $K_{\Gamma}\left(b_{x, z}, \infty\right) \leq A$. Also, we have $K_{\Gamma}\left(b_{y, z}, \infty\right) \geq A>0$ because of $\delta_{\Gamma}\left(b_{y, z}\right) \geq\left(2 \kappa^{2}\right)^{-1} A_{5}^{-1} r_{1}$ by Proposition 3.1. Hence (3.8) holds in this case.

Case 2: $r<r_{1}$. Let $\zeta \in \partial \Gamma$ be a point such that $\delta_{\Gamma}(z)=|z-\zeta|$. Then $\left|b_{x, z}-\zeta\right| \leq$ $\left|b_{x, z}-z\right|+|z-\zeta| \leq(2 \kappa+1) A_{5}^{-1} r \leq A_{1} r$. We take $w \in \Gamma \cap S\left(\zeta, A_{1} r\right)$ with $\delta_{\Gamma}(w) \approx r$. Note that

$$
\begin{aligned}
\left|w-b_{y, z}\right| & \leq|w-\zeta|+|\zeta-z|+\left|z-b_{y, z}\right| \leq\left(A_{1}+(1+\kappa) A_{5}^{-1}\right) r \\
& \leq A \min \left\{\delta_{\Gamma}(w), \delta_{\Gamma}\left(b_{y, z}\right)\right\}
\end{aligned}
$$

since $\delta_{\Gamma}\left(b_{y, z}\right) \geq\left(2 \kappa^{2}\right)^{-1} A_{5}^{-1} r$ by Proposition 3.1. Hence Lemmas 2.3 and 2.2 yield that

$$
K_{\Gamma}\left(b_{x, z}, \infty\right) \leq A K_{\Gamma}(w, \infty) \approx K_{\Gamma}\left(b_{y, z}, \infty\right) .
$$

Hence (3.8) holds. Thus the theorem is proved.

Theorem 3.5. There exists a constant $A$ depending only on $\Gamma$ such that for $x, y \in \Gamma$,

$$
\frac{G_{\Gamma}(x, y) K_{\Gamma}(y, \infty)}{K_{\Gamma}(x, \infty)} \leq A|x-y|^{2-n}
$$

Proof. We may assume by (2.5) that $x, y \in \Gamma \cap B(1)$. Let $b \in \mathcal{B}(x, y)$. Then we have by Theorem 3.2

$$
\frac{G_{\Gamma}(x, y) K_{\Gamma}(y, \infty)}{K_{\Gamma}(x, \infty)} \approx\left(\frac{K_{\Gamma}(y, \infty)}{K_{\Gamma}(b, \infty)}\right)^{2}|x-y|^{2-n} .
$$

To obtain (3.9), it suffices to show $K_{\Gamma}(y, \infty) / K_{\Gamma}(b, \infty) \leq A$. This can be proved by the similar way as in the proof of Theorem 3.4. For the reader's convenience, we give a proof. Let $r=A_{5} \max \left\{|x-y|, \delta_{\Gamma}(y)\right\}$, where $A_{5}=3 \kappa A_{1}^{-1}$. If $r \geq r_{1}$, then $\delta_{\Gamma}(b) \geq$ $\left(2 \kappa^{2}\right)^{-1} A_{5}^{-1} r_{1}$ by Proposition 3.1, and therefore $K_{\Gamma}(y, \infty) \leq A K_{\Gamma}(b, \infty)$. Suppose in the sequel that $r<r_{1}$. Let $\xi \in \partial \Gamma$ be a point such that $\delta_{\Gamma}(b)=|b-\xi|$, and take $w \in \Gamma \cap S\left(\xi, A_{1} r\right)$ with $\delta_{\Gamma}(w) \approx r$. Then

$$
|y-\xi| \leq|y-b|+\delta_{\Gamma}(b) \leq 2|y-b|+\delta_{\Gamma}(y) \leq(2 \kappa+1) A_{5}^{-1} r \leq A_{1} r
$$

and

$$
\begin{aligned}
|b-w| & \leq|b-\xi|+|\xi-w| \leq|b-y|+\delta_{\Gamma}(y)+|\xi-w| \\
& \leq\left((\kappa+1) A_{5}^{-1}+A_{1}\right) r \leq A \min \left\{\delta_{\Gamma}(b), \delta_{\Gamma}(w)\right\} .
\end{aligned}
$$

Hence we obtain from Lemmas 2.3 and 2.2

$$
K_{\Gamma}(y, \infty) \leq A K_{\Gamma}(w, \infty) \approx K_{\Gamma}(b, \infty) .
$$

Thus the theorem is proved.

Remark 3.6. From the proof of Theorem 3.5, it follows in general that

$$
\max \left\{K_{\Gamma}(x, \infty), K_{\Gamma}(y, \infty)\right\} \leq A K_{\Gamma}(b, \infty) \quad \text { for } x, y \in \Gamma \text { and } b \in \mathcal{B}(x, y) .
$$

The following $3 \mathrm{G}$ inequality has been studied widely in many bounded domains, but it may be unknown in cones. 
Corollary 3.7 (Classical 3G inequality). There exists a constant A depending only on $\Gamma$ such that for $x, y, z \in \Gamma$,

$$
\frac{G_{\Gamma}(x, y) G_{\Gamma}(y, z)}{G_{\Gamma}(x, z)} \leq A\left(|x-y|^{2-n}+|y-z|^{2-n}\right) .
$$

Proof. This follows immediately from Theorems 3.4 and 3.5.

The following two lemmas will be used in the subsequent sections, so we prove them here.

Lemma 3.8. There exists a constant $A$ depending only on $\Gamma$ such that for $x, y \in \Gamma$,

$$
K_{\Gamma}(x, \infty) K_{\Gamma}(y, \infty) \leq A \max \{|x|,|y|\}^{n-2+2 \alpha} G_{\Gamma}(x, y),
$$

where $\alpha \geq 0$ is the constant in Lemma 2.5.

Proof. Let $b \in \mathcal{B}(x, y)$ and $R=\max \{|x|,|y|\}$. Since $|b| \leq|b-x|+|x| \leq \kappa|x-y|+|x| \leq$ $(2 \kappa+1) R$, we have by Lemma 2.5

$$
\frac{|x-y|^{2-n}}{K_{\Gamma}(b, \infty)^{2}} \geq A \frac{R^{2-n}}{R^{2 \alpha}} .
$$

Thus the lemma follows from Theorem 3.2.

Lemma 3.9. Let $r>0$ and $R>0$. Then there exists a constant $A$ depending only on $r, R$ and $\Gamma$ such that for $x, y \in \Gamma \cap B(R)$ with $|x-y| \geq r$,

$$
G_{\Gamma}(x, y) \leq A K_{\Gamma}(x, \infty) K_{\Gamma}(y, \infty) .
$$

Proof. Let $\alpha \geq 0$ and $\theta$ be as in Lemma 2.5, and let $\beta \geq 1$ be as in (2.6). Then we have

$$
K_{\Gamma}(b, \infty)=|b|^{\alpha} \theta(b /|b|) \geq A|b|^{\alpha-\beta} \delta_{\Gamma}(b)^{\beta} \quad \text { for } b \in \mathcal{B}(x, y) .
$$

Since $|b| \leq(2 \kappa+1) R$ and $\delta_{\Gamma}(b) \geq \kappa^{-1}|x-y| \geq \kappa^{-1} r$, it follows from Theorem 3.2 that

$$
G_{\Gamma}(x, y) \leq A(r, R) K_{\Gamma}(x, \infty) K_{\Gamma}(y, \infty),
$$

where $A(r, R)$ is a positive constant depending on $r$ and $R$. Thus the lemma is proved.

Remark 3.10. If $\alpha \geq 1=\beta$, then $K_{\Gamma}(b, \infty) \geq A \max \{|x|,|y|,|x-y|\}^{\alpha-1}|x-y|$ by (3.11) and Proposition 3.1, and therefore we have

$$
G_{\Gamma}(x, y) \leq A \frac{K_{\Gamma}(x, \infty) K_{\Gamma}(y, \infty)}{\max \{|x|,|y|,|x-y|\}^{2(\alpha-1)}|x-y|^{n}} \quad \text { for } x, y \in \Gamma,
$$

where the constant $A$ depends only on $\Gamma$. If $\alpha<1=\beta$, then $|b| \leq(2 \kappa+1) \max \{|x|,|y|\}$, and so $K_{\Gamma}(b, \infty) \geq A \max \{|x|,|y|\}^{\alpha-1}|x-y|$. Therefore we have

$$
G_{\Gamma}(x, y) \leq A \frac{K_{\Gamma}(x, \infty) K_{\Gamma}(y, \infty)}{\max \{|x|,|y|\}^{2(\alpha-1)}|x-y|^{n}} \quad \text { for } x, y \in \Gamma,
$$

where the constant $A$ depends only on $\Gamma$. 


\section{Generalized Cranston-McConnell inequality and extended Kato class}

In [8], Cranston and McConnell proved that if $\Omega$ is a domain in $\mathbb{R}^{2}$ with the finite volume $\operatorname{vol}(\Omega)$, then there exists a constant $A$ such that

$$
\sup _{x, h} \frac{1}{h(x)} \int_{\Omega} G_{\Omega}(x, y) h(y) d y \leq A \operatorname{vol}(\Omega),
$$

where the supremum is taken over all $x \in \Omega$ and all positive harmonic functions $h$ in $\Omega$. In general, if $\Omega$ has infinite volume, then the left hand side of the above inequality diverges. We consider the following generalization. In the rest of this section, we suppose that $\Gamma$ is a uniform cone in $\mathbb{R}^{n}$ with $n \geq 3$. By $\mathcal{U}_{+}(\Gamma)$, we denote the class of all positive superharmonic functions in $\Gamma$. We say that a measure $\nu$ on $\Gamma$ enjoys the generalized Cranston-McConnell inequality if

$$
\|\nu\|_{C M}:=\sup _{x \in \Gamma, u \in \mathcal{U}_{+}(\Gamma)} \frac{1}{u(x)} \int_{\Gamma} G_{\Gamma}(x, y) u(y) d \nu(y)<+\infty .
$$

See [1] for investigations and related topics of the generalized Cranston-McConnell inequality, and see reference therein. We give necessary and sufficient conditions for $\nu$ to satisfy the generalized Cranston-McConnell inequality. To simplify the notation, we write

$$
H_{\Gamma}(x, y)=\frac{G_{\Gamma}(x, y) K_{\Gamma}(y, \infty)}{K_{\Gamma}(x, \infty)} \quad \text { for } x, y \in \Gamma
$$

and set

$$
\|\nu\|_{H}=\sup _{x \in \Gamma} \int_{\Gamma} H_{\Gamma}(x, y) d \nu(y) .
$$

The following theorem means that $\nu$ enjoys the generalized Cranston-McConnell inequality if (4.1) holds only for $u=K_{\Gamma}(\cdot, \infty)$.

Theorem 4.1. Let $\nu$ be a measure on $\Gamma$. The following statements are equivalent:

(i) $\|\nu\|_{C M}<+\infty$;

(ii) $\|\nu\|_{H}<+\infty$;

(iii) $\|\nu\|_{G}:=\sup _{x, z \in \Gamma} \int_{\Gamma} \frac{G_{\Gamma}(x, y) G_{\Gamma}(y, z)}{G_{\Gamma}(x, z)} d \nu(y)<+\infty$.

Moreover, $\|\nu\|_{C M} \approx\|\nu\|_{H} \approx\|\nu\|_{G}$, where the constants of comparisons depend only on $\Gamma$.

Proof. If (i) holds, then we obtain (ii) by taking $u=K_{\Gamma}(\cdot, \infty)$ in (4.1). If (ii) holds, then we have by Theorem 3.4

$$
\int_{\Gamma} \frac{G_{\Gamma}(x, y) G_{\Gamma}(y, z)}{G_{\Gamma}(x, z)} d \nu(y) \leq A \int_{\Gamma}\left\{H_{\Gamma}(x, y)+H_{\Gamma}(z, y)\right\} d \nu(y) .
$$

Hence (iii) follows. We finally show that (iii) implies (i). By assumption, we have

$$
\int_{\Gamma} G_{\Gamma}(x, y) G_{\Gamma}(y, z) d \nu(y) \leq\|\nu\|_{G} G_{\Gamma}(x, z) \quad \text { for } x, z \in \Gamma .
$$

Let $G_{\Gamma} \mu$ be a Green potential in $\Gamma$ of a measure $\mu$. It then follows from Fubini's theorem that

$$
\int_{\Gamma} G_{\Gamma}(x, y) G_{\Gamma} \mu(y) d \nu(y) \leq\|\nu\|_{G} G_{\Gamma} \mu(x)
$$


Since every positive superharmonic function can be approximated by an increasing sequence of Green potentials, the monotone convergence theorem yields (i). Thus the theorem is proved.

The following is an immediate consequence of Theorems 3.5 and 4.1 .

Corollary 4.2. If $\nu$ is a measure on $\Gamma$ satisfying

$$
\sup _{x \in \Gamma} \int_{\Gamma}|x-y|^{2-n} d \nu(y)<+\infty,
$$

then $\nu$ enjoys the generalized Cranston-McConnell inequality.

As another consequence, we obtain the following.

Corollary 4.3. Let $\nu$ be a measure on $\Gamma$ such that $\|\nu\|_{H}<+\infty$. Then for each $R>0$,

$$
\int_{\Gamma \cap B(R)} K_{\Gamma}(y, \infty) d \nu(y)<+\infty .
$$

Proof. Let $R>1$. Then Lemma 3.8 with $x=x_{0}$ and Theorem 4.1 yield that

$$
\int_{\Gamma \cap B(R)} K_{\Gamma}(y, \infty) d \nu(y) \leq A \int_{\Gamma} G_{\Gamma}\left(x_{0}, y\right) d \nu(y) \leq A\|\nu\|_{C M}<+\infty,
$$

and thus the corollary follows.

We now introduce an extended Kato class.

Definition 4.4. We say that a measure $\nu$ on $\Gamma$ belongs to the extended Kato class $\mathcal{K}(\Gamma)$ if $\nu$ fulfills the following properties:

$$
\begin{aligned}
& \lim _{r \rightarrow 0}\left(\sup _{x \in \Gamma} \int_{\Gamma \cap B(x, r)} H_{\Gamma}(x, y) d \nu(y)\right)=0, \\
& \lim _{R \rightarrow+\infty}\left(\sup _{x \in \Gamma} \int_{\Gamma \backslash B(R)} H_{\Gamma}(x, y) d \nu(y)\right)=0 .
\end{aligned}
$$

We also say that a Borel measurable function $f$ in $\Gamma$ belongs to the extended Kato class if the measure $d \nu=|f| d y$ belongs to $\mathcal{K}(\Gamma)$.

The classical Kato class $\mathcal{K}_{0}(\Gamma)$ is the set of all measures $\nu$ on $\Gamma$ satisfying (4.2) and (4.3) with the Newtonian kernel $|x-y|^{2-n}$ instead of $H_{\Gamma}(x, y)$. By Theorem 3.5, we easily see $\mathcal{K}_{0}(\Gamma) \subset \mathcal{K}(\Gamma)$.

Proposition 4.5. If $\nu$ is a measure on $\Gamma$ such that $\nu \in \mathcal{K}(\Gamma)$, then $\|\nu\|_{H}<+\infty$. Moreover, for each $R>0$,

$$
\int_{\Gamma \cap B(R)} K_{\Gamma}(y, \infty)^{2} d \nu(y)<+\infty .
$$

Proof. Let $R>0$. By the definition of $\mathcal{K}(\Gamma)$, there exist positive numbers $r_{2}$ and $R_{2}$ such that

$$
\sup _{x \in \Gamma} \int_{\Gamma \cap B\left(x, r_{2}\right)} H_{\Gamma}(x, y) d \nu(y) \leq 1 \quad \text { and } \quad \sup _{x \in \Gamma} \int_{\Gamma \backslash B\left(R_{2}\right)} H_{\Gamma}(x, y) d \nu(y) \leq 1 .
$$


Since $\Gamma \cap B\left(R_{2}\right)$ is covered by a finite sequence of balls $\left\{B\left(x_{j}, r_{2}\right)\right\}_{j=1}^{m}$ with $x_{j} \in \Gamma \cap$ $B\left(R_{2}\right)$, it follows from Lemma 3.8 and (4.4) that

$$
\int_{\Gamma \cap B\left(R_{2}\right)} K_{\Gamma}(y, \infty)^{2} d \nu(y) \leq A \sum_{j=1}^{m} \int_{\Gamma \cap B\left(x_{j}, r_{2}\right)} H_{\Gamma}\left(x_{j}, y\right) d \nu(y)<+\infty .
$$

Therefore we have by Lemma 3.9

$$
\sup _{x \in \Gamma} \int_{\Gamma \cap B\left(R_{2}\right) \backslash B\left(x, r_{2}\right)} H_{\Gamma}(x, y) d \nu(y)<+\infty .
$$

This, together with (4.4), yields that $\|\nu\|_{H}<+\infty$.

Corollary 4.6. If $\nu$ be a measure on $\Gamma$ such that $\nu \in \mathcal{K}(\Gamma)$, then for each $z_{0} \in \bar{\Gamma}$,

$$
\lim _{r \rightarrow 0}\left(\sup _{x \in \Gamma} \int_{\Gamma \cap B\left(z_{0}, r\right)} H_{\Gamma}(x, y) d \nu(y)\right)=0 .
$$

Proof. Let $\varepsilon>0$. Since $\nu \in \mathcal{K}(\Gamma)$, there exist positive numbers $r_{3}$ and $R_{3}$ such that

$$
\sup _{x \in \Gamma} \int_{\Gamma \cap B\left(x, r_{3}\right)} H_{\Gamma}(x, y) d \nu(y) \leq \varepsilon \quad \text { and } \quad \sup _{x \in \Gamma} \int_{\Gamma \backslash B\left(R_{3}\right)} H_{\Gamma}(x, y) d \nu(y) \leq \varepsilon .
$$

Let $r>0$ and $x \in \Gamma$. Then we have by Lemma 3.9

$$
\begin{aligned}
\int_{\Gamma \cap B\left(z_{0}, r\right)} H_{\Gamma}(x, y) d \nu(y) & \leq 2 \varepsilon+\int_{\Gamma \cap B\left(z_{0}, r\right) \cap B\left(R_{3}\right) \backslash B\left(x, r_{3}\right)} H_{\Gamma}(x, y) d \nu(y) \\
& \leq 2 \varepsilon+A \int_{\Gamma \cap B\left(z_{0}, r\right) \cap B\left(R_{3}\right)} K_{\Gamma}(y, \infty)^{2} d \nu(y) .
\end{aligned}
$$

In veiw of Proposition 4.5, we obtain (4.5).

In the sequel, let $\alpha \geq 0$ and $\theta$ be as in Lemma 2.5. We give examples for the strictness of the inclusion $\mathcal{K}_{0}(\Gamma) \subset \mathcal{K}(\Gamma)$ when $\partial \Gamma \cap S(1)$ has a $C^{1,1}$-boundary. Note in this case that $\theta(z) \approx \delta_{\Gamma}(z)$ for $z \in \Gamma \cap S(1)$ and that if $\tau \geq 1$, then for $\xi \in \partial \Gamma \cap S(1)$ and $r>0$,

$$
\int_{\Gamma \cap B(\xi, r)} \delta_{\Gamma}(y)^{-\tau} d y=+\infty .
$$

Since $\mathcal{K}_{0}(\Gamma) \subset L^{1}(\Gamma \cap B(2))$, we see in each example below that $V \in \mathcal{K}(\Gamma) \backslash \mathcal{K}_{0}(\Gamma)$ if $1 \leq p<2<q$.

Example 4.7. Suppose that $\alpha \geq 1$ and $\theta(z) \approx \delta_{\Gamma}(z)$, and let

$$
V(y)=(1+|y|)^{p-q} \delta_{\Gamma}(y)^{-p} .
$$

Then $V \in \mathcal{K}(\Gamma)$ if and only if $p<2<q$.

Proof. Note that $V(y) \approx(1+|y|)^{p-q}|y|^{p(\alpha-1)} K_{\Gamma}(y, \infty)^{-p}$. We first show the sufficiency, so we assume that $p<2<q$. Let $r>0$ and $x \in \Gamma$. If $p<0$, then $H_{\Gamma}(x, y) V(y) \leq$ $A|x-y|^{2-n}$. Therefore

$$
\int_{\Gamma \cap B(x, r)} H_{\Gamma}(x, y) V(y) d y \leq A r^{2},
$$

and so $V$ satisfies (4.2). We consider the case $p \geq 0$. It follows from Theorem 3.2 and (3.10) that for $b \in \mathcal{B}(x, y)$,

$$
H_{\Gamma}(x, y) V(y) \leq A K_{\Gamma}(b, \infty)^{-p}|x-y|^{2-n}(1+|y|)^{p-q}|y|^{p(\alpha-1)} .
$$


Since $\alpha \geq 1$, it follows from Proposition 3.1 that $K_{\Gamma}(b, \infty) \approx|b|^{\alpha-1} \delta_{\Gamma}(b) \geq A|y|^{\alpha-1} \mid x-$ $y \mid$. Therefore we have

$$
\int_{\Gamma \cap B(x, r)} H_{\Gamma}(x, y) V(y) d y \leq A \int_{\Gamma \cap B(x, r)}|x-y|^{2-n-p} d y \leq A r^{2-p} .
$$

Hence $V$ satisfies (4.2). We next show that $V$ satisfies (4.3). Observe from Remark 3.10 and Lemma 2.5 that

$$
H_{\Gamma}(x, y) V(y) \leq A \frac{|y|^{2-q}}{|x-y|^{n}} .
$$

Let $R>0$. Then it follows from the above observation that

$$
\begin{aligned}
\sup _{x \in \Gamma} \int_{\Gamma \backslash B(R)} H_{\Gamma}(x, y) V(y) d y & \leq A\left(r^{2-p}+I_{1}+I_{2}+I_{3}+I_{4}\right) \\
& \leq A\left(r^{2-p}+R^{2-q}+\sup _{|x| \geq 2^{-1} R}|x|^{2-q} \log \frac{3|x|}{r}\right),
\end{aligned}
$$

where

$$
\begin{aligned}
& I_{1}=\sup _{|x| \leq 2^{-1} R} \int_{\Gamma \backslash(B(R) \cup B(x, r))} \frac{|y|^{2-q}}{|x-y|^{n}} d y, \\
& I_{2}=\sup _{|x| \geq 2^{-1} R} \int_{\left\{|y| \leq 2^{-1}|x|\right\} \backslash(B(R) \cup B(x, r))} \frac{|y|^{2-q}}{|x-y|^{n}} d y, \\
& I_{3}=\sup _{|x| \geq 2^{-1} R} \int_{\left\{2^{-1}|x| \leq|y| \leq 2|x|\right\} \backslash(B(R) \cup B(x, r))} \frac{|y|^{2-q}}{|x-y|^{n}} d y, \\
& I_{4}=\sup _{|x| \geq 2^{-1} R} \int_{\{2|x| \leq|y|\} \backslash(B(R) \cup B(x, r))} \frac{|y|^{2-q}}{|x-y|^{n}} d y .
\end{aligned}
$$

Hence $V$ satisfies (4.3), and thus $V \in \mathcal{K}(\Gamma)$. Conversely, we suppose that $V \in \mathcal{K}(\Gamma)$. Note that $H_{\Gamma}(x, y) \approx|x-y|^{2-n} \geq A \delta_{\Gamma}(x)^{2-n}$ for $y \in B\left(x, 2^{-1} \delta_{\Gamma}(x)\right)$. Hence we have

$$
H_{\Gamma}(x, y) V(y) \geq A \delta_{\Gamma}(x)^{2-n-p}(1+|x|)^{p-q} \text { for } y \in B\left(x, 2^{-1} \delta_{\Gamma}(x)\right) .
$$

Let $r>0$, and let $x \in \Gamma \cap S(1)$ be a point such that $\delta_{\Gamma}(x) \leq r$. Since $B\left(x, 2^{-1} \delta_{\Gamma}(x)\right) \subset$ $\Gamma \cap B(x, r)$, it follows that

$$
\int_{\Gamma \cap B(x, r)} H_{\Gamma}(x, y) V(y) d y \geq A \delta_{\Gamma}(x)^{2-p} .
$$

Therefore it must be $p<2$ to satisfy (4.2). Let $R>0$. Similarly, if $x=\rho x_{0}$ with $\rho \geq 2 R$, then $B\left(x, 2^{-1} \delta_{\Gamma}(x)\right) \subset \Gamma \backslash B(R)$, and so

$$
\int_{\Gamma \backslash B(R)} H_{\Gamma}(x, y) V(y) d y \geq A \rho^{2-p}(1+\rho)^{p-q} .
$$

Hence it must be $q>2$ to satisfy (4.3).

Example 4.8. Suppose that $0 \leq \alpha<1$ and $\theta(z) \approx \delta_{\Gamma}(z)$, and let

$$
V(y)=(1+|y|)^{\alpha p-q}|y|^{p(1-\alpha)} \delta_{\Gamma}(y)^{-p} .
$$

Then $V \in \mathcal{K}(\Gamma)$ if and only if $p<2<q$. 
Proof. Note that $V(y) \approx(1+|y|)^{\alpha p-q} K_{\Gamma}(y, \infty)^{-p}$. We first assume that $p<2<q$. Let $0<r<1$ and $x \in \Gamma$. If $p<0$, then $H_{\Gamma}(x, y) V(y) \leq A|x-y|^{2-n}$. Therefore

$$
\int_{\Gamma \cap B(x, r)} H_{\Gamma}(x, y) V(y) d y \leq A r^{2},
$$

and so $V$ satisfies (4.2). We consider the case $p \geq 0$. If $y \in B(x, r)$, then $1+|y| \approx 1+|x|$, and so

$$
K_{\Gamma}(b, \infty) \approx|b|^{\alpha-1} \delta_{\Gamma}(b) \geq A(1+|x|)^{\alpha-1}|x-y| \quad \text { for } b \in \mathcal{B}(x, y) .
$$

It follows from Theorem 3.2 and (3.10) that

$$
\int_{\Gamma \cap B(x, r)} H_{\Gamma}(x, y) V(y) d y \leq A(1+|x|)^{p-q} \int_{\Gamma \cap B(x, r)}|x-y|^{2-n-p} d y \leq A r^{2-p} .
$$

Hence $V$ satisfies (4.2). We next show that $V$ satisfies (4.3). Observe from Remark 3.10 and Lemma 2.5 that

$$
H_{\Gamma}(x, y) V(y) \leq A \frac{|y|^{2 \alpha-q}}{\max \{|x|,|y|\}^{2(\alpha-1)}|x-y|^{n}}
$$

Let $R>0$. Then it follows from the above observation that

$$
\begin{aligned}
\sup _{x \in \Gamma} \int_{\Gamma \backslash B(R)} H_{\Gamma}(x, y) V(y) d y & \leq A\left(r^{2-p}+I_{1}+I_{2}^{\prime}+I_{3}+I_{4}\right) \\
& \leq A\left(r^{2-p}+R^{2-q}+\sup _{|x| \geq 2^{-1} R}|x|^{2-q} \log \frac{3|x|}{r}\right),
\end{aligned}
$$

where $I_{1}, I_{3}$ and $I_{4}$ are as in (4.7), and

$$
I_{2}^{\prime}=\sup _{|x| \geq 2^{-1} R} \int_{\left\{|y| \leq 2^{-1}|x|\right\} \backslash(B(R) \cup B(x, r))} \frac{|y|^{2 \alpha-q}}{|x|^{2(\alpha-1)}|x-y|^{n}} d y .
$$

Hence $V$ satisfies (4.3), and thus $V \in \mathcal{K}(\Gamma)$. Conversely, we suppose that $V \in \mathcal{K}(\Gamma)$. Observe that

$$
H_{\Gamma}(x, y) V(y) \geq A \delta_{\Gamma}(x)^{2-n-p}(1+|x|)^{\alpha p-q}|x|^{p(1-\alpha)} \quad \text { for } y \in B\left(x, 2^{-1} \delta_{\Gamma}(x)\right) .
$$

Let $r>0$ and let $x \in \Gamma \cap S(1)$ be a point such that $\delta_{\Gamma}(x) \leq r$. Then $B\left(x, 2^{-1} \delta_{\Gamma}(x)\right) \subset$ $\Gamma \cap B(x, r)$, and so

$$
\int_{\Gamma \cap B(x, r)} H_{\Gamma}(x, y) V(y) d y \geq A \delta_{\Gamma}(x)^{2-p} .
$$

Therefore it must be $p<2$ to satisfy (4.2). Let $R>0$. Similarly, if $x=\rho x_{0}$ with $\rho \geq 2 R$, then $B\left(x, 2^{-1} \delta_{\Gamma}(x)\right) \subset \Gamma \backslash B(R)$, and so

$$
\int_{\Gamma \backslash B(R)} H_{\Gamma}(x, y) V(y) d y \geq A \rho^{2-\alpha p}(1+\rho)^{\alpha p-q} .
$$

Therefore it must be $q>2$ to satisfy (4.3). 


\section{Nonlinear Schrödinger problem}

In this section, we consider the following nonlinear Schrödinger problem:

$$
\left\{\begin{array}{l}
\Delta u-\mu u=f(\cdot, u) \text { in } \Gamma \text { (in the sense of distributions), } \\
u>0 \text { in } \Gamma \\
u=0 \text { on } \partial_{r} \Gamma
\end{array}\right.
$$

where $\partial_{r} \Gamma$ denotes the set of all Dirichlet regular points of $\partial \Gamma$. We assume the following condition on $\mu$ and $f$. By $|\mu|$, we denote the total variational measure of a signed measure $\mu$.

(P1) $\mu$ is a signed measure on $\Gamma$ such that $|\mu| \in \mathcal{K}(\Gamma)$ and $\||\mu|\|_{H}<2^{-1}$.

(P2) $f$ is a Borel measurable function in $\Gamma \times(0,+\infty)$ such that $f(x, \cdot)$ is continuous in $(0,+\infty)$ for each $x \in \Gamma$.

(P3) $|f(x, t)| \leq t \psi(x, t)$ for $(x, t) \in \Gamma \times(0,+\infty)$, where $\psi$ is some non-negative measurable function in $\Gamma \times(0,+\infty)$ such that for each $x \in \Gamma, \psi(x, \cdot)$ is non-decreasing in $(0,+\infty)$ and $\lim _{t \rightarrow 0+} \psi(x, t)=0$.

(P4) $\varphi(x)=\psi\left(x, K_{\Gamma}(x, \infty)\right)$ belongs to $\mathcal{K}(\Gamma)$.

Theorem 5.1. Let $\Gamma$ be a uniform cone in $\mathbb{R}^{n}$ with $n \geq 3$. Assume that $\mu$ and $f$ satisfy (P1)-(P4). Then the problem (5.1) has infinitely many continuous solutions. More precisely, there exists a positive constant $\lambda_{0}$ such that for each $\lambda \in\left(0, \lambda_{0}\right]$, the problem (5.1) has a continuous solution u satisfying that

$$
\frac{2\left(1-2\||\mu|\|_{H}\right)}{3-2\|\| \mu \mid \|_{H}} \lambda K_{\Gamma}(x, \infty) \leq u(x) \leq \frac{4}{3-2\||\mu|\|_{H}} \lambda K_{\Gamma}(x, \infty) \quad \text { for } x \in \Gamma,
$$

and

$$
\lim _{x \rightarrow \infty} \frac{u(x)}{K_{\Gamma}(x, \infty)}=\lambda
$$

The proof is based on the Schauder's fixed point argument. In the sequel, we suppose that $\Gamma$ is a uniform cone in $\mathbb{R}^{n}$ with $n \geq 3$ and that $\mu$ and $f$ satisfy (P1)-(P4). Let $C(\Gamma)$ denote the space of all bounded continuous functions in $\Gamma$ endowed with the uniform norm $\|\cdot\|_{\infty}$, and let

$$
C_{0}(\bar{\Gamma} \cup\{\infty\})=\left\{w \in C(\bar{\Gamma} \cup\{\infty\}): \lim _{x \rightarrow \infty} w(x)=0\right\} .
$$

We also write

$$
C_{1}(\Gamma)=\{w \in C(\Gamma): 0<w(x) \leq 1 \text { for } x \in \Gamma\} .
$$

For $w \in C_{1}(\Gamma)$, we define

$$
F w(x)=\int_{\Gamma} H_{\Gamma}(x, y) w(y) d \mu(y)+\frac{1}{K_{\Gamma}(x, \infty)} \int_{\Gamma} G_{\Gamma}(x, y) f\left(y, w(y) K_{\Gamma}(y, \infty)\right) d y .
$$

In what follows, we write $d \nu(y)=d|\mu|(y)+\varphi(y) d y$ to simplify the notation. Note from (P1) and (P4) that $\nu \in \mathcal{K}(\Gamma)$.

Lemma 5.2. The class $\left\{F w: w \in C_{1}(\Gamma)\right\}$ is equicontinuous in $\bar{\Gamma} \cup\{\infty\}$. Moreover, $\left\{F w: w \in C_{1}(\Gamma)\right\} \subset C_{0}(\bar{\Gamma} \cup\{\infty\})$. 
Proof. Let $z_{0} \in \bar{\Gamma}$ and $\delta>0$. Let $x_{1}, x_{2} \in \Gamma \cap B\left(z_{0}, 2^{-1} \delta\right)$. It follows from (P3) and $w \leq 1$ that

$$
\begin{aligned}
& \left|F w\left(x_{1}\right)-F w\left(x_{2}\right)\right| \\
& \leq 2 \sup _{x \in \Gamma} \int_{\Gamma \backslash B\left(\delta^{-1}\right)} H_{\Gamma}(x, y) d \nu(y)+2 \sup _{x \in \Gamma} \int_{\Gamma \cap B\left(z_{0}, \delta\right)} H_{\Gamma}(x, y) d \nu(y) \\
& \quad+\int_{\Gamma \cap B\left(\delta^{-1}\right) \backslash B\left(z_{0}, \delta\right)}\left|\frac{G_{\Gamma}\left(x_{1}, y\right)}{K_{\Gamma}\left(x_{1}, \infty\right)}-\frac{G_{\Gamma}\left(x_{2}, y\right)}{K_{\Gamma}\left(x_{2}, \infty\right)}\right| K_{\Gamma}(y, \infty) d \nu(y) .
\end{aligned}
$$

By (4.3) and Corollary 4.6, the first two quantities of the right hand side are bounded by $\varepsilon$ whenever $\delta$ is sufficiently small. If $z_{0} \in \Gamma$, then $B\left(z_{0}, \delta\right) \subset \Gamma$ for sufficiently small $\delta$, and so $G_{\Gamma}(\cdot, y) / K_{\Gamma}(\cdot, \infty)$ is continuous in $B\left(z_{0}, 2^{-1} \delta\right)$ whenever $y \in \Gamma \backslash B\left(z_{0}, \delta\right)$. If $z_{0} \in \partial \Gamma$, then $G_{\Gamma}(\cdot, y) / K_{\Gamma}(\cdot, \infty)$ can be extended continuously to $\bar{\Gamma} \cap B\left(z_{0}, \delta_{1}\right)$ whenever $y \in \Gamma \backslash B\left(z_{0}, \delta\right)$ and $\delta_{1} \in(0, \delta)$ is sufficiently small (cf. [2, Theorem 2]). In any cases, it follows from Corollary 4.3 and Lebesgue's convergence theorem that, as $\left|x_{1}-x_{2}\right| \rightarrow 0$,

$$
\int_{\left(\Gamma \cap B\left(\delta^{-1}\right)\right) \backslash B\left(z_{0}, \delta\right)}\left|\frac{G_{\Gamma}\left(x_{1}, y\right)}{K_{\Gamma}\left(x_{1}, \infty\right)}-\frac{G_{\Gamma}\left(x_{2}, y\right)}{K_{\Gamma}\left(x_{2}, \infty\right)}\right| K_{\Gamma}(y, \infty) d \nu(y) \rightarrow 0 .
$$

Hence $F w$ is continuous in $\bar{\Gamma}$ uniformly for $w$. We next consider $z_{0}=\infty$. Let $R>0$. Then

$$
|F w(x)| \leq \int_{\Gamma \cap B(R)} H_{\Gamma}(x, y) d \nu(y)+\sup _{z \in \Gamma} \int_{\Gamma \backslash B(R)} H_{\Gamma}(z, y) d \nu(y) .
$$

By (4.3), the second term of the right hand side is bounded by $\varepsilon$ whenever $R$ is sufficiently large. Note from Corollary 3.3 and (2.4) that

$$
H_{\Gamma}(x, y) \leq A R^{2-n-2 \alpha} K_{\Gamma}(y, \infty)^{2} \text { for } x \in \Gamma \backslash B(2 R) \text { and } y \in \Gamma \cap B(R) .
$$

It follows from Proposition 4.5 and Lebesgue's convergence theorem that $|F w(x)| \rightarrow 0$ uniformly for $w \in F$ as $x \rightarrow \infty$. Thus $\left\{F w: w \in C_{1}(\Gamma)\right\}$ is equicontinuous in $\bar{\Gamma} \cup\{\infty\}$, and is contained in $C_{0}(\bar{\Gamma} \cup\{\infty\})$.

Let $\||\mu|\|_{H}<2^{-1}$. For $\lambda>0$, we define

$$
\mathcal{W}_{\lambda}=\left\{w \in C(\bar{\Gamma} \cup\{\infty\}): \frac{2\left(1-2\||\mu|\|_{H}\right)}{3-2\||| \mu \mid\|_{H}} \lambda \leq w(x) \leq \frac{4}{3-2\||\mu|\|_{H}} \lambda \text { for } x \in \Gamma\right\} .
$$

Obviously, $\mathcal{W}_{\lambda}$ is the non-empty bounded closed convex set in $C(\bar{\Gamma} \cup\{\infty\})$. Note that if $\lambda \leq 2^{-1}$, then $\mathcal{W}_{\lambda} \subset C_{1}(\Gamma)$. We define the operator $T_{\lambda}$ on $\mathcal{W}_{\lambda}$ by

$$
T_{\lambda} w(x)=\lambda-F w(x) \quad \text { for } x \in \Gamma,
$$

and write $T_{\lambda}\left(\mathcal{W}_{\lambda}\right)=\left\{T_{\lambda} w: w \in \mathcal{W}_{\lambda}\right\}$

Lemma 5.3. There exists a positive constant $\lambda_{0}$ such that if $0<\lambda \leq \lambda_{0}$, then $T_{\lambda}\left(\mathcal{W}_{\lambda}\right) \subset$ $\mathcal{W}_{\lambda}$. Moreover, $T_{\lambda}\left(\mathcal{W}_{\lambda}\right)$ is relatively compact in $C(\bar{\Gamma} \cup\{\infty\})$.

Proof. Let $0<\lambda \leq 2^{-1}$ and let $w \in \mathcal{W}_{\lambda}$. Since $F w \in C(\bar{\Gamma} \cup\{\infty\})$ by Lemma 5.2, we have $T_{\lambda} w \in C(\bar{\Gamma} \cup\{\infty\})$. It suffices to show that there exists a positive constant $\lambda_{0} \leq 2^{-1}$ such that if $0<\lambda \leq \lambda_{0}$, then

$$
\frac{2\left(1-2\|\| \mu \mid \|_{H}\right)}{3-2\||\mu|\|_{H}} \lambda \leq T_{\lambda} w(x) \leq \frac{4}{3-2\||\mu|\|_{H}} \lambda \quad \text { for } x \in \Gamma .
$$


Let $0<\tau \leq 1$ and define

$$
\Psi_{\tau}(x)=\int_{\Gamma} H_{\Gamma}(x, y) \psi\left(y, \tau K_{\Gamma}(y, \infty)\right) d y \quad \text { for } x \in \Gamma .
$$

As in the proof of Lemma 5.2, we see that $\Psi_{\tau} \in C_{0}(\bar{\Gamma} \cup\{\infty\})$. Moreover, for each $x \in \Gamma$, it follows from (P3) that the function $\tau \mapsto \Psi_{\tau}(x)$ is non-decreasing and $\Psi_{\tau}(x) \rightarrow 0$ as $\tau \rightarrow 0$. Therefore we have by Dini's theorem

$$
\lim _{\tau \rightarrow 0}\left(\sup _{x \in \Gamma} \Psi_{\tau}(x)\right)=0 .
$$

We take $0<\tau_{0} \leq 2^{-1}$ so that $\sup _{x \in \Gamma} \Psi_{\tau_{0}}(x) \leq 4^{-1}\left(1-2\||\mu|\|_{H}\right)$. Let $\lambda_{0}=4^{-1}(3-$ $\left.2\||\mu|\|_{H}\right) \tau_{0}$ and let $0<\lambda \leq \lambda_{0}$. Then $4\left(3-2\|\| \mu \mid \|_{H}\right)^{-1} \lambda \leq \tau_{0}$, and so it follows from (P3) that for $x \in \Gamma$,

$$
\left|T_{\lambda} w(x)-\lambda\right|=|F w(x)| \leq \frac{4 \lambda\||| \mu \mid\|_{H}}{3-2\||| \mu \mid\|_{H}}+\frac{4 \lambda}{3-2\||\mu|\|_{H}} \Psi_{\tau_{0}}(x) \leq \frac{1+2\||\mu|\|_{H}}{3-2\||\mu|\|_{H}} \lambda .
$$

Hence we obtain (5.4), and thus $T_{\lambda}\left(\mathcal{W}_{\lambda}\right) \subset \mathcal{W}_{\lambda}$. Since $T_{\lambda}\left(\mathcal{W}_{\lambda}\right)$ is uniformly bounded and is equicontinuous in $\bar{\Gamma} \cup\{\infty\}$ by Lemma 5.2, it follows from Ascoli-Arzelà's theorem that $T_{\lambda}\left(\mathcal{W}_{\lambda}\right)$ is relatively compact in $C(\bar{\Gamma} \cup\{\infty\})$. Thus the lemma is proved.

Lemma 5.4. Let $0<\lambda \leq \lambda_{0}$. Then $T_{\lambda}$ is continuous on $\mathcal{W}_{\lambda}$.

Proof. Let $\left\{w_{j}\right\}$ be a sequence in $\mathcal{W}_{\lambda}$ converging to $w \in \mathcal{W}_{\lambda}$ with respect to $\|\cdot\|_{\infty}$. Then, for each $x \in \Gamma$, it follows from (P1)-(P4), Proposition 4.5 and Lebesgue's convergence theorem that, as $j \rightarrow+\infty$,

$$
\begin{aligned}
& \int_{\Gamma} H_{\Gamma}(x, y)\left|w_{j}(y)-w(y)\right| d|\mu|(y) \rightarrow 0, \\
& \frac{1}{K_{\Gamma}(x, \infty)} \int_{\Gamma} G_{\Gamma}(x, y)\left|f\left(y, w_{j}(y) K_{\Gamma}(y, \infty)\right)-f\left(y, w(y) K_{\Gamma}(y, \infty)\right)\right| d y \rightarrow 0 .
\end{aligned}
$$

Hence $T_{\lambda} w_{j}$ converges pointwisely to $T_{\lambda} w$ in $\Gamma$ as $j \rightarrow+\infty$. Since $T_{\lambda}\left(\mathcal{W}_{\lambda}\right)$ is relatively compact in $C(\bar{\Gamma} \cup\{\infty\})$, the pointwise convergence implies the uniform convergence. Therefore $\left\|T_{\lambda} w_{j}-T_{\lambda} w\right\|_{\infty} \rightarrow 0$ as $j \rightarrow+\infty$. Thus $T_{\lambda}$ is continuous on $\mathcal{W}_{\lambda}$.

Let us prove Theorem 5.1.

Proof of Theorem 5.1. Let $0<\lambda \leq \lambda_{0}$, where $\lambda_{0}$ is the positive constant in Lemma 5.3. Note again that $\mathcal{W}_{\lambda}$ is the non-empty bounded closed convex set in $C(\bar{\Gamma} \cup\{\infty\})$. Since $T_{\lambda}$ is compact mapping from $\mathcal{W}_{\lambda}$ into itself, it follows from Schauder's fixed point theorem that there exists $w \in \mathcal{W}_{\lambda}$ such that $w=T_{\lambda} w=\lambda-F w$, that is, for $x \in \Gamma$,

$$
w(x)=\lambda-\int_{\Gamma} H_{\Gamma}(x, y) w(y) d \mu(y)-\frac{1}{K_{\Gamma}(x, \infty)} \int_{\Gamma} G_{\Gamma}(x, y) f\left(y, w(y) K_{\Gamma}(y, \infty)\right) d y .
$$

Let $u(x)=w(x) K_{\Gamma}(x, \infty)$. Then $u>0$ in $\Gamma$, and $u=0$ on $\partial_{r} \Gamma$ since $K_{\Gamma}(\cdot, \infty)$ is so. Also, we have for $x \in \Gamma$,

$$
\frac{2\left(1-2\||\mu|\|_{H}\right)}{3-2\||| \mu \mid\|_{H}} \lambda K_{\Gamma}(x, \infty) \leq u(x) \leq \frac{4}{3-2\||\mu|\|_{H}} \lambda K_{\Gamma}(x, \infty)
$$

and

$$
u(x)=\lambda K_{\Gamma}(x, \infty)-\int_{\Gamma} G_{\Gamma}(x, y) u(y) d \mu(y)-\int_{\Gamma} G_{\Gamma}(x, y) f(y, u(y)) d y .
$$


Since $K_{\Gamma}(\cdot, \infty)$ is harmonic in $\Gamma$, we see that $u$ is a distributional solution to $\Delta u-\mu u=$ $f(\cdot, u)$ in $\Gamma$. Finally, since $F w \in C_{0}(\bar{\Gamma} \cup\{\infty\})$, it follows that

$$
\lim _{x \rightarrow \infty} \frac{u(x)}{K_{\Gamma}(x, \infty)}=\lambda-\lim _{x \rightarrow \infty} F w(x)=\lambda .
$$

Thus the proof of Theorem 5.1 is complete.

\section{REFERENCES}

[1] H. Aikawa, Norm estimate of Green operator, perturbation of Green function and integrability of superharmonic functions, Math. Ann. 312 (1998), no. 2, 289-318.

[2] _ Boundary Harnack principle and Martin boundary for a uniform domain, J. Math. Soc. Japan 53 (2001), no. 1, 119-145.

[3] H. Aikawa, K. Hirata and T. Lundh, Martin boundary points of a John domain and unions of convex sets, J. Math. Soc. Japan (to appear).

[4] H. Aikawa and T. Lundh, The 3G inequality for a uniformly John domain, Kodai Math. J. (to appear).

[5] I. Bachar, H. Mâagli and M. Zribi, Estimates on the Green function and existence of positive solutions for some polyharmonic nonlinear equations in the half space, Manuscripta Math. 113 (2004), no. 3, 269-291.

[6] K. Bogdan, Sharp estimates for the Green function in Lipschitz domains, J. Math. Anal. Appl. 243 (2000), no. 2, 326-337.

[7] M. Cranston, E. Fabes and Z. Zhao, Conditional gauge and potential theory for the Schrödinger operator, Trans. Amer. Math. Soc. 307 (1988), no. 1, 171-194.

[8] M. Cranston and T. R. McConnell, The lifetime of conditioned Brownian motion, Z. Wahrsch. Verw. Gebiete 65 (1983), no. 1, 1-11.

[9] F. W. Gehring and B. G. Osgood, Uniform domains and the quasihyperbolic metric, J. Analyse Math. 36 (1979), 50-74.

[10] Ü. Kuran, On NTA-conical domains, J. London Math. Soc. (2) 40 (1989), no. 3, 467-475.

[11] Q. S. Zhang and Z. Zhao, Singular solutions of semilinear elliptic and parabolic equations, Math. Ann. 310 (1998), no. 4, 777-794.

[12] Z. Zhao, On the existence of positive solutions of nonlinear elliptic equations - a probabilistic potential theory approach, Duke Math. J. 69 (1993), no. 2, 247-258.

Department of Mathematics, Hokkaido University, Sapporo 060-0810, Japan

E-mail address: hirata@math.sci.hokudai.ac.jp 